INPLASY

PROTOCOL

To cite: Cai et al. Comparative safety of PARP inhibitors in cancer: A network metaanalysis. Inplasy protocol 202130018. doi:

10.37766/inplasy2021.3.0018

Received: 06 March 2021

Published: 06 March 2021

Corresponding author: Zhaolun Cai

caizhaolun@foxmail.com

Author Affiliation:

Department of Gastrointestinal

Surgery, West China Hospital,

Sichuan University

Support: None.

Review Stage at time of this submission: Data analysis.

Conflicts of interest:

None declared.

\section{Comparative safety of PARP inhibitors in cancer: A network meta-analysis}

Cai, Z1; Liu, C2; Chang, C3; Shen, C4; Yin, Y5; Yin, X6; Jiang, Z7; Zhao, Z8; Mu, M97 Cao, D10; Zhang, L11; Zhang, ${ }^{12}$.

Review question / Objective: The objective of the current study is to conduct comprehensive drug-based network meta-analysis (mixed treatment comparisons) of the comparative safety of approved PRAP inhibitors, with the aim of providing a complete toxicity profile and a safety ranking of approved PRAP inhibitors.

Condition being studied: Poly ADP ribose polymerase (PARP) inhibitors have become one of the most important breakthroughs in cancer treatment, especially, but not only, for patients with BRCA-associated tumors.

Information sources: We searched the PubMed, Embase (Ovid), and the Cochrane Central Register of Controlled Trials (CENTRAL) for relevant RCTs published from inception to 30st December 2020.

INPLASY registration number: This protocol was registered with the International Platform of Registered Systematic Review and Meta-Analysis Protocols (INPLASY) on 06 March 2021 and was last updated on 06 March 2021 (registration number INPLASY202130018).

\section{INTRODUCTION}

Review question / Objective: The objective of the current study is to conduct comprehensive drug-based network metaanalysis (mixed treatment comparisons) of the comparative safety of approved PRAP inhibitors, with the aim of providing a complete toxicity profile and a safety ranking of approved PRAP inhibitors.

Condition being studied: Poly ADP ribose polymerase (PARP) inhibitors have become one of the most important breakthroughs in 
cancer treatment, especially, but not only, for patients with BRCA-associated tumors.

\section{METHODS}

Participant or population: Patients with cancer according to the histologic examination.

Intervention: RCTs comparing any two or three of the following approved treatments or different doses of the same PARP inhibitors were included: fluzoparib, olaparib, rucaparib, niraparib and talazoparib.

\section{Comparator: Placebo.}

Study designs to be included: RCTs.

Eligibility criteria: The inclusion criteria complied with PICOS (population, intervention, comparators, outcomes and study design) described above.

Information sources: We searched the PubMed, Embase (Ovid), and the Cochrane Central Register of Controlled Trials (CENTRAL) for relevant RCTs published from inception to 30st December 2020.

Main outcome(s): 1) Serious adverse events: An adverse event that results in death, is life-threatening, requires inpatient hospitalization or extends a current hospital stay, results in an ongoing or significant incapacity or interferes substantially with normal life functions, or causes a congenital anomaly or birth defect. Medical events that do not result in death, are not life-threatening, or do not require hospitalization may be considered serious adverse events if they put the participant in danger or require medical or surgical intervention to prevent one of the results listed above. 2) Discontinuations due to adverse events.

Additional outcome(s): 1) Interruption of treatment due to adverse event. 2) Dose reduction due to adverse event. 3) Specific adverse events: nausea, diarrhea decreased appetite, vomiting, constipation, abdominal pain, dyspepsia, dysgeusia, fatigue or asthenia, dizziness, insomnia, headache, dyspnea, nasopharyngitis, cough, arthralgia, back pain, anemia, thrombocytopenia or platelet count decreased, neutropenia.

Quality assessment / Risk of bias analysis: Two reviewers assessed the risk of bias of included studies with the Cochrane Handbook's Risk of Bias assessment tool independently and resolved disagreements by discussion.

Strategy of data synthesis: We generated plots depicting the network geometry using Stata version 15.0 (StataCorp) and performed a network meta-analysis using GeMTC.

Subgroup analysis: NA.

Sensitivity analysis: NA.

Country(ies) involved: China.

Keywords: cancer, PARP inhibitors, SAE.

Contributions of each author:

Author 1 - Zhaolun Cai.

Author 2 - Chunyu Liu.

Author 3 - Chen Chang.

Author 4 - Chaoyong Shen.

Author 5 - Yuan Yin.

Author 6 - Xiaonan Yin.

Author 7 - Zhiyuan Jiang.

Author 8 - Zhou Zhao.

Author 9 - Mingchun Mu.

Author 10 - Dan Cao.

Author 11 - Lingli Zhang.

Author 12 - Bo Zhang. 